\title{
Pattern of Skin Disease Among Clients Attending Dermatologic Clinic at Finote Selam Hospital, West Gojjam Zone, Amhara Region, Ethiopia, 2017
}

\author{
Sintayehu Worku Aynalem \\ Department of Dermatology, Finote Selam Hospital, Finote Selam, Ethiopia
}

Email address:

worku.sintayehu@yahoo.com

To cite this article:

Sintayehu Worku Aynalem. Pattern of Skin Disease Among Clients Attending Dermatologic Clinic at Finote Selam Hospital, West Gojjam Zone, Amhara Region, Ethiopia, 2017. American Journal of Health Research. Vol. 5, No. 6, 2017, pp. 178-182.

doi: 10.11648/j.ajhr.20170506.13

Received: June 29, 2017; Accepted: July 21, 2017; Published: December 3, 2017

\begin{abstract}
Background: Skin diseases and their complications are a significant burden on the health system of many nations. Skin problem account significant public health issue in developing countries and affects $20-30 \%$ of the general population. Pattern of skin diseases vary from region to region which are influenced by various factors and the consequent morbidities have a profound effect on individual and the community. Therefore, the objective of this study was to assess the pattern of skin disease among clients attending dermatologic outpatient clinic at Finote Selam Hospital, West Gojjam Zone, Amhara Region, North West Ethiopia, 2017. Methods and Materials: Hospital based cross-sectional study was conducted and took all individuals during study period at Finote Selam Hospital dermatologic unit. Structured questionnaire was used for interviewing and data was collected using an interviewer administered questionnaire. The data was entered and analysis was done by SPSS version 16.0. Descriptive statistics was done. The magnitude of non infectious skin disease was $62 \%$. The majorities 90 $(29.5 \%)$ of skin diseases were eczema. Fungal infections $35(11.5 \%)$ were the most common infectious skin problem. Farmers (25.8\%) were the most affected group. Nearly two third (63.2\%) of skin diseases were at urban. The magnitude of non infectious skin disease was relatively higher than infectious skin disease. Eczema was highly prevailing skin diseases. Therefore, early diagnosis and treatment of skin disease, aware dermatologic disease for health professional, available necessary medication and Educate the patients to consult the health professionals when they have skin problem were recommended.
\end{abstract}

Keywords: Pattern of Skin Disease, Finote Selam Hospital, Infectious, Non Infectious Skin Disease

\section{Introduction}

\section{Background}

Skin diseases and their complications are a significant burden on the health system of many nations. AS World Health Organization report on mortality rate of the skin disease accounted that 20,000 in Sub-Saharan Africa. Skin problem account for a significant public health issue in developing countries and affects $20-30 \%$ of the general population. And Pattern of skin diseases varies from region to region which are influenced by various factors and the consequent morbidities have a profound effect on individual and the community $[1,2]$.

Skin disease causes a huge burden in the global health. Skin conditions were the 4th leading cause of nonfatal burden expressed as disability in 2010. In developed country pruritus accounts highest prevalence while scabies is the lowest. But in developing country especially in sub-Saharan African infectious like bacterial, fungal, and viral skin disease are predominant. Skin problem measures both non fatal and fatal health burden [3, 4]. Which are not recognized as a major public health problem in developing countries, despite World Health Organization (WHO) estimates that 21$87 \%$ of the general population in developing countries has skin problem. Problems with the skin are among the main reasons for seeking care, accounting for up to $24 \%$ of primary care visits, and are one of the most common causes 
of morbidity. Infections and infestations make up the majority of skin disease in many Sub-Saharan African countries accounted that $85 \%$ in Tanzania, $78 \%$ in Malawi, $71.5 \%$ in Ethiopia, and $40.1 \%$ in Uganda $[3,4,5,6]$.

\section{Objectives}

\subsection{General Objective}

Assess the pattern of skin disease among clients attending dermatologic outpatient clinic at Finote Selam Hospital, West Gojjam, Amhara, and North West Ethiopia.

\subsection{Specific Objectives}

(a). To determine pattern of skin disease among clients attending dermatologic clinic at Finote Selam Hospital.

(b). To assess the prevalence of infectious and non infectious skin disease among clients attending dermatologic clinic at Finote Selam Hospital.

\section{Methodology}

\subsection{Study Area, Study Design and Period}

Institution based cross -sectional study design was conducted from Feb-1-30, 2017 at Finote Selam Hospital.

\subsection{Population}

\subsubsection{Source Population}

All patients with skin problem at Finote Selam Hospital.

\subsubsection{Study Population}

All dermatologic patients on dermatology Clinic at Finote Selam Hospital during

Study period.

\subsubsection{Sample Population}

All dermatologic patients at dermatology Clinic in Finote Selam Hospital during

Study period.

\subsubsection{Inclusion and Exclusion Criteria}

All clients who visit dermatologic unit at Finote Selam Hospital during the

Study period except who has ambitious clinical diagnosis of skin problem.

\subsection{Sample Size Determination and Sampling Technique}

\subsubsection{Sample Size}

The sample size was determined by using a single population proportion formula and considering the following assumptions: A 95\% confidence level, margin of error (0.05) and average prevalence of skin problem in developing country $(p=0.25)$. These parameters are substituted in the following single population proportion formula.

$$
n=\frac{(Z \alpha / 2)^{2} P(1-P)}{d^{2}}
$$

Where:

n-the desired sample size

P- Average prevalence of skin problem in developing country $(25 \%)$.

$\mathrm{Z} \alpha / 2$ - critical value for normal distribution at $95 \%$ confidence level which equals to $1.96(\mathrm{z}$ value at $=0.05)$

$\mathrm{d}$ - The margin of error taken as 0.05

So the formula yields 288 sample size and adding 10\% (29 clients) for non-respondent. Therefore the total sample size is 317.

\subsubsection{Sampling Technique}

A convenience sampling method was used. All clients attending dermatologic unit were examined for clinical diagnose of skin problem and interviewed.

\subsection{Study Variables}

\subsubsection{Dependent Variables}

Pattern of skin disease

\subsubsection{Independent Variables}

Socio demographic characters: Age, Sex, Marital status, residence, occupation, educational status, and religion.

Clinical characteristics: Medical problem (RVI, DM, and HTN)

\subsection{Data Collection Tools and Procedures}

\subsubsection{Data Collection Tools}

Data was collected using structured questionnaire which were adopted from different literatures based on objectives of the study. The questionnaire was prepared in English.

\subsubsection{Data Collection Procedures}

Data was collected by interviewer administered questionnaire and physical examination. The data was collected by principal investigator (MSc in Dermatology) and assistant three BSc Nurses. At the end of the day principal investigator checked daily activity of data regarding the completion of the questionnaires, clarity of responses and proper coding of the respondents.

\subsection{Data Management and Analysis}

The collected data was checked for completeness, range and logical sequence of responses. The completed questionnaire checked for completeness, consistency and coded by the principal investigator and data encoder. Data was entered in to SPSS version 16.0 software package for analysis. Data was presented by frequency, tables and graphs. Descriptive statistics was computed to determine the pattern of skin disease.

\subsection{Quality Control}

To ensure data quality, every day at the end of the data 
collection discussion was made with the assistant data collectors. If problems encounter, find the solution. The completeness of the questionnaire was checked before data entry. Since there are no similar facilities which manage dermatologic cases around the study area so pretest was done at dermatology clinic at Finote Selam Hospital prior to actual data collection. Participants in this pretest were not included in the study. The principal investigator checked collected data for completeness and corrective measures was taken accordingly. And also three days training was given to assistant data collectors.

\subsection{Ethical Consideration}

Ethical clearance was obtained from the chief Executive Officer of the Finote Selam Hospital. Accordingly, permission letter was secured from CEO at Finote Selam Hospital. In addition all of the study participants were informed about the purpose of the study and oral/verbal consent was obtained before interview, examine and also ensure during each step of data collection. Respondents were notified that they had the right to refuse or terminate at any point of the interview and the information provide by each respondent was kept confidential. Patient identification variables such as name, house number, phone number were not used in the study. This study was not inflicting harm on or exposes the patients to unnecessary risk. The responses given by the provider for the questionnaire was very essential to know the pattern of skin problem. And also helps for the successful accomplishment of the study.

\section{Result}

Socio-demographic Characteristics

Out of 317 dermatologic cases at dermatologic OPD were planned to participate in this study, 302 were included in the analysis making the response rate of $95.3 \%$. The mean $( \pm$ SD) age of the respondent was $26( \pm 14.75)$ years. Majority $(32.5 \%)$ of the respondents were present in age group of (21$30 \mathrm{yrs})$. One hundred fifty nine $(52.6 \%)$ were males while $288(95.4 \%)$ of the respondents were orthodox by religion. Concerning the educational status of patient, $61.3 \%$ had attended formal school, out of which $18.5 \%$ had accomplished above twelve. Around half of patients were married; farmers and students account $25.8 \%$ and $24.5 \%$ respectively. Nearly two third (63.2\%) of them were urban by place of residence (Table 1).

Table 1. Socio-demographic characteristics of patients in Finote Selam Hospital at dermatology OPD, Amhara Regional Stat, North West Ethiopia, 2017.

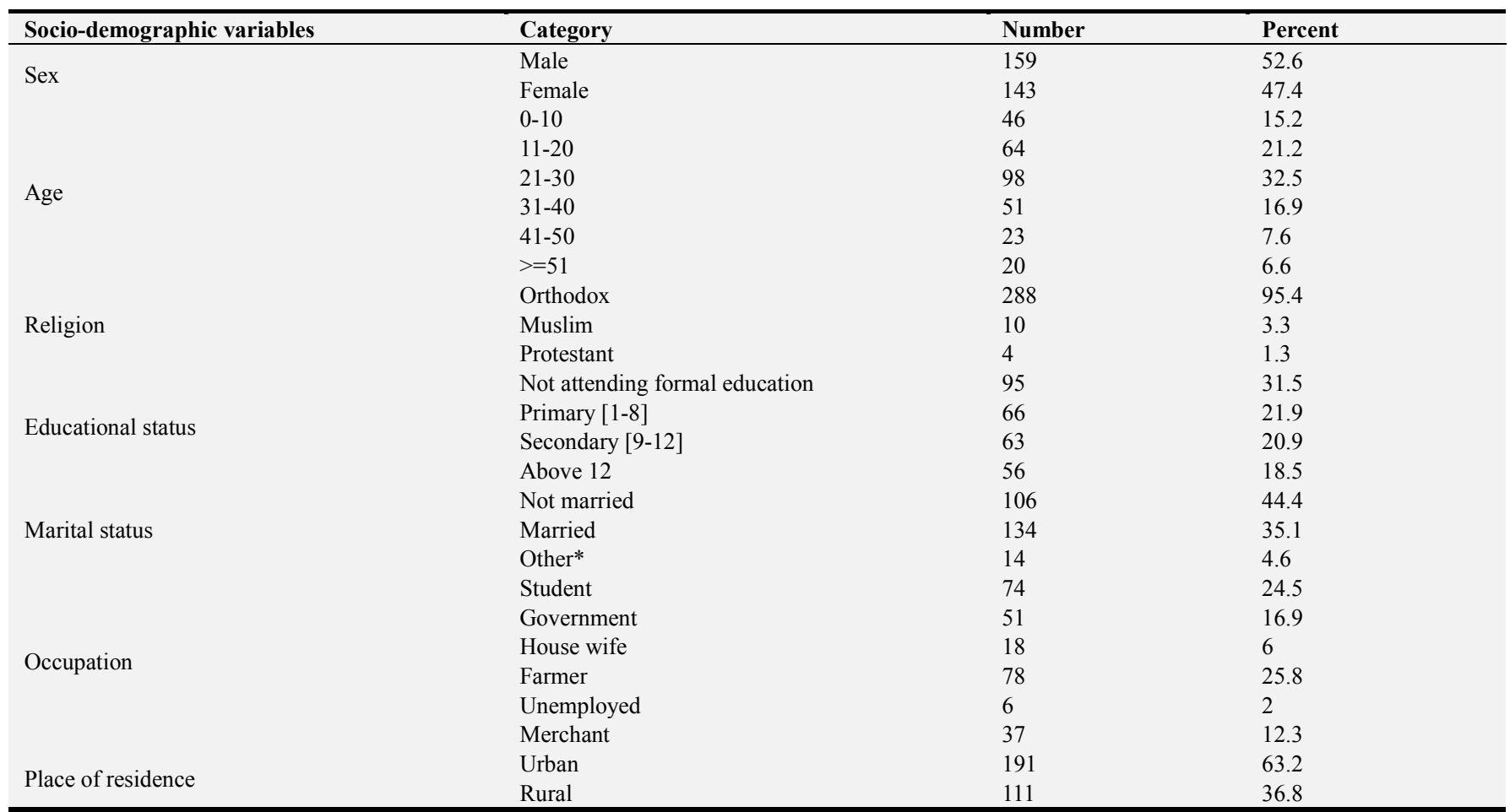

*Divorce, Widowed

\section{Clinical Characteristics}

Among the pattern of skin problem eczema was the most common finding which accounted that $90(29.5 \%)$, followed by pigmentary skin disease $36(11.9 \%)$. Non infectious and infectious skin disease accounted that $(62.9 \% \mathrm{v} \quad 37.1 \%)$. Infectious skin disease like fungal, bacterial, cutaneous leishmaniasis, viral, and infestation accounted that 35 (11.6\%),
$26(8.6 \%), 19(6.3 \%), 18(6.1 \%)$ respectively. Fungal skin disease was the most common infectious skin dermatoses with predominance of tinea capitis which accounted that $11(3.6 \%)$ while on eczema, atopic dermatitis accounted that $38(12.6 \%)$. Other non infectious skin disease such as papulosquamous, pilosebaceous, and urticaria showed that 23(7.6\%), 16(5.3\%), and $14(4.6 \%)$ respectively (Table 2 ). 
Table 2. Clinical characteristics of pattern of skin disease in Finote Selam Hospital at dermatologic OPD, Amhara Regional Stat, North West Ethiopia, 2017.

\begin{tabular}{|c|c|c|c|}
\hline Clinical variables & Category & Number & Percent \\
\hline \multirow{6}{*}{ Ezema $90(29.5 \%)$} & Atopic dermatitis & 38 & 42.2 \\
\hline & Contact dermatitis & 19 & 21.1 \\
\hline & Lichen simplex chronicus & 14 & 15.6 \\
\hline & Seborrhic dermatitis & 11 & 12.2 \\
\hline & Nummular eczema & 6 & 6.7 \\
\hline & Other $^{* *}$ & 2 & 2.2 \\
\hline \multirow{3}{*}{ Pigmentary $36(11.9 \%)$} & Vitiligo & 27 & 75 \\
\hline & Melasma & 5 & 13.9 \\
\hline & Erytheromelanosis & 4 & 11.1 \\
\hline \multirow{5}{*}{ Fungal Infection 35 (11.6\%) } & Tinea capitis & 11 & 31.4 \\
\hline & Cadidal infection & 10 & 28.6 \\
\hline & Tineacorporis & 7 & 20 \\
\hline & Onycomycosis & 4 & 11.4 \\
\hline & Tineapedis & 3 & 8.6 \\
\hline \multirow{5}{*}{ Bacterial infectin $26(8.6 \%)$} & Impetigo & 11 & 42.3 \\
\hline & Leprosy & 6 & 23 \\
\hline & Furuncle & 4 & 15.4 \\
\hline & Folliculitis & 4 & 15.4 \\
\hline & SSSS & 1 & 3.9 \\
\hline \multirow{2}{*}{ Papulosquamous $23(7.6 \%)$} & Lichen planus & 12 & 52.2 \\
\hline & Psoriasis & 11 & 47.8 \\
\hline \multirow{2}{*}{ Cutaneous leishmaniasis 19 (6.3\%) } & Mucocutaneous & 11 & 57.9 \\
\hline & Localized & 8 & 42.1 \\
\hline \multirow{5}{*}{ Viral infection $18(8.6 \%)$} & Chicken pox & 5 & 27.8 \\
\hline & Herpes zoster & 4 & 22.2 \\
\hline & Molluscum & 4 & 22.2 \\
\hline & Wart & 3 & 16.7 \\
\hline & Herpes simplex & 2 & 11.1 \\
\hline \multirow{3}{*}{ Pilosebaceous 16 (5.3\%) } & Acne & 13 & 81.2 \\
\hline & Rosacea & 2 & 12.5 \\
\hline & Perioral dermatitis & 1 & 6.3 \\
\hline \multirow{2}{*}{ Urticaria $14(4.6 \%)$} & Acute & 8 & 57.1 \\
\hline & Chronic & 6 & 42.9 \\
\hline \multirow{2}{*}{ Infestation $14(4.6 \%)$} & Scabies & 13 & 92.9 \\
\hline & Pediculosis & 1 & 7.1 \\
\hline Other $* * *$ & & 11 & 3.6 \\
\hline Infectious & & 112 & 37.1 \\
\hline Non infectious & & 190 & 62.9 \\
\hline
\end{tabular}

** Stasis dermatitis, Pityriasis Alba, ${ }^{* * * K e l o i d, ~ p e m p h i g u s ~ F o l l a c e o u s, ~ D i s c o i d ~ l u p u s ~ e r t h e m a t o u s ~}$

\section{Discussion}

The current study attempted to determine the prevalence of infectious and non infectious skin disease with the pattern of skin problem.

In this study, the majority (62.9\%) of skin disease was non infectious. This was slightly higher than Indian study which accounted that $54.3 \%$ [7] and lower than study conducted at Black lion, which showed that $74.5 \%$ [12]; whereas study done at Nigeria accounted that $14.8 \%$ [10]. It was very low on the aspect of non infectious skin disease as compare to this study. For this discrepancy, it might be sociodemography and Nigerian study was done at specifically on diabetic patient, so diabetic patients are venerable to infectious rather than non infectious skin dermatoses.

Concerning on the clinical characteristic of skin disease, the prevalence of the eczema in this study was $29.5 \%$, which was similar to study conducted at Black lion (25.5\%) [12]. While there was discrepancy on study done at India accounted that 7.1\% [7] and Saudi Arabia (15.8\%) [8]. The possible reason for the discrepancy could be sociodemographic or it might be the study design, especially Saudi Arabia study was retrospective while this study was crosssectional. Regarding to infectious skin disease the prevalence of bacterial infection in this study accounted that $8.5 \%$ which was similar to study conducted at Tanzania showed that $7 \%$ [11] and Cameron 5.2\% [9]. But which was lower than Nigerian study $16.9 \%$ [10]. Pigmentary skin disease at Cameron accounted that $4.8 \%$, whereas in this study showed that $11.9 \%$, which was higher than Cameron study, so the possible reason could be socio-demography, and socioeconomy. There was also study conducted at Black Lion, papulosquamous accounted that $11.4 \%$ [12], while in this study which showed that $7.6 \%$.

Regarding to the socio-demographic characteristics of the study conducted at different area, female respondent accounted that in India 51.5\% [7], Saudi Arabia 52.8\% [8], Cameron 50\% [9], Tanzania 56.7\% [11], Nigeria 54.2\% [10]. But in this study female respondent accounted that $47.4 \%$ which was lower than all study above. So the possible reason 
could be study design, socio-economic variation and low level of awareness about the disease. Concerning comparison between urban and rural areas higher prevalence was found in urban areas at India [7] which is similar to this study by which two third of pattern of skin disease were from urban places. Majority $(32.5 \%)$ of the dermatological disorders were present in age group of (21-30 yrs) in this study. which was similar to study conducted at Saudi Arabia (21-30 yrs) [8] and Black lion (21-40 yrs) [12]. Also study conducted at India on occupational status of the respondent, students accounted that $23.5 \%$ [7], which was almost similar to this study $(24.5 \%)$, while lower than study at Cameron (37.5\%) [9].

\section{Conclusion and Recommendation}

\subsection{Conclusion}

This study had clearly defined the different types of skin disease. Study reveals that non infectious skin disease was more common. Eczema was the most common non infectious skin disease. Fungal and Bacterial infections were common among infectious skin diseases.

\subsection{Recommendation}

To regional health bureau and stakeholder

(a). Should strengthen its attention for dermatologic diseases control while designing and implementing health programs and strategies as well as aware dermatologic disease for health professional

To health professionals

(a). Early diagnosis and treatment of skin disease

(b). Available necessary medication

(c). Educate the patients to consult the health professionals when they have skin problem

(d). Aware the community on the environmental allergens and educate skin disease can treated as other medical problem

To researcher

(a). Assess the factors associated with skin disease using stronger study design

\section{Acknowledgements}

I would like to acknowledge Finote Selam Hospital Chief
Excusive Officer to assist me ideally and financially from the beginning of my title selection up to final thesis. And my gratitude goes to the data collectors and respondents without them this thesis would not have been realized.

\section{References}

[1] World Health Organization, 2005 /accessed on Feb 2017 GC/.

[2] Williams. H. Epidemiology of skin diseases. Blackwell Science; 2004: 6-7.

[3] Vos T, Flaxman D, Naghavi M, et al. systematic analysis for the Global Burden of Disease Study. Lancet 2010; 380:216396.

[4] Lozano R, Naghavi M, Foreman K et al. systematic analysis for the Global Burden of Disease Study. Lancet 2010; 380:2095-128.

[5] Jenny. H, Karen. M, Art. P, et al. Dermatology and HIV/AIDS in Africa. Journal of global infectious disease, 2011 Jul-Sep; $3(3): 275-280$.

[6] Gibbs S. Skin disease and socioeconomic conditions in rural Africa: Tanzania. Int J Dermatol. 1996; 35:633-9.

[7] DrBaijayanti. B, DrJhuma. S, DrNirmalya. M. et al. The Pattern of Dermatological Disorders among Patients Attending the Skin O. P. D of A Tertiary Care Hospital in Kolkata, India. Journal of Dental and Medical Sciences 2013 Feb; 3(4):04-09.

[8] Hani. A, Shobaili. A. The pattern of skin diseases in the Qassim region of Saudi Arabia. Annals of Saudi Medicine 2010 Nov-Dec; 30(6): 448-453.

[9] Anne. C, Zoung. K, Earnest. N et al. The spectrum of skin diseases in a rural setting in Cameroon. BMC Dermatol 2012; 12: 7 .

[10] Ezejiofor O, Onayemi O, Olasode A, et al. Patterns of dermatological disorders among diabetics. Egyptian Dermatology Online Journal Dec 2013: 9 (2); 1.

[11] Yassin. M, Mgonda. M, and Pauline N. The burden of coexisting dermatological disorders and their tendency of being overlooked among patients admitted to muhimbili national hospital in Dar es Salaam, Tanzania. Mgonda and Chale BMC Dermatology 2011, 11:8.

[12] Shibeshi D. Pattern of skin diseases at the University Teaching Hospital, Addis Ababa, Ethiopia. Int J Dermatol 2000; 39: $822-825$. 